\title{
Associations between energy drink consumption and school attendance, academic attainment, and problem behaviour: A cross-sectional and longitudinal analysis
}

\author{
Gareth Richards, Andrew P. Smith
}

Published Online November 25, 2016

Autism Research Centre, Department of Psychiatry, School of Clinical Medicine, University of Cambridge, Cambridge, UK (G Richards MSc); and Centre for Occupational \& Health Psychology, School of Psychology, Cardiff University, Cardiff, UK (G Richards, Prof A P Smith $\mathrm{PhD})$

Correspondence to: Mr Gareth Richards, Autism Research Centre, University of Cambridge, Douglas House, Cambridge CB2 8AH, UK gvr22@medschl.cam.ac.uk

\begin{abstract}
Background Some investigators have claimed that caffeinated energy drinks negatively impact children's behaviour and academic attainment. The present study therefore investigated associations between energy drink use and school attendance, English and mathematics attainment, and the occurrence of behavioural sanctions (eg, detention).
\end{abstract}

Methods Two cross-sections of data were collected at two timepoints (December, 2012; June, 2013). All children $(n=3071)$ attending three secondary schools in the South West of England were invited to participate, and no specific inclusion or exclusion criteria were imposed. Questionnaires were used to record dietary and lifestyle information, and data relating to demography and school performance were acquired through the School Information Management System. Cross-sectional, crosslag, and change score analyses were initially conducted with $\chi^{2}$ tests. Binary logistic regression was then used to control for additional aspects of diet, demography, and lifestyle. The Cardiff University School of Psychology Ethics Committee granted ethics approval (EC.12.09.11.3187). All children and their parents gave written informed consent. The research was conducted in accordance with the Declaration of Helsinki.

Findings 2030 children responded to the questionnaire at the first timepoint and 2307 at the second. Cross-sectional analyses showed that energy drink consumption was negatively associated with school performance. The effects appeared to be dose dependent. For example, relative to never using the products, each frequency of consumption was associated with below average English attainment at the second timepoint: "once a month" (odds ratio $1 \cdot 6,95 \%$ CI 1·2-2.1), "once or twice a week" $(1 \cdot 9,1 \cdot 3-2 \cdot 6)$, "most days $(3-6)$ " $(2 \cdot 1,1 \cdot 3-3 \cdot 5)$, "every day" $(2 \cdot 3,1 \cdot 1-4 \cdot 9)$. Crosslag analyses yielded similar results, though the effects were not significant at the multivariate level. Increasing frequency of energy drink usage between the two timepoints was predictive of not increasing in English and mathematics attainment, and with increasing in number of behavioural sanctions incurred. This last effect remained significant after covariates were controlled for (odds ratio 1.9, 95\% CI 1.3$2 \cdot 7, \mathrm{p}=0 \cdot 0003)$.

Interpretation Energy drink consumption was consistently associated with low school performance. Although only some effects remained significant at the 
multivariate level, the results as a whole suggest that energy drinks might be a cause for concern. Intervention studies are now required to better determine the nature of the associations observed.

Funding The Waterloo Foundation (grant number 503692). The funder had no role in the study design or data analysis.

\section{Contributors}

APS designed the study, acquired funding, and supervised the running of the project. GR analysed and interpreted the data and wrote the abstract. APS then revised the abstract for important intellectual content, and both authors read and approved the abstract before submission. GR had full access to all data in the study and had final responsibility for the decision to submit for publication.

\section{Declaration of interests}

Both authors received grants from The Waterloo Foundation during the conduct of the study. 